\title{
Poly(ADP-ribose) polymerase 2 contributes to neuroinflammation and neurological dysfunction in mouse experimental autoimmune encephalomyelitis
}

\author{
Amit Kamboj ${ }^{1,2}$, Ping Lu ${ }^{1,2}$, Michael B Cossoy ${ }^{3}$, Jillian L Stobart ${ }^{1,2}$, Brian A Dolhun ${ }^{1,2}$, Tiina M Kauppinen', \\ Gilbert de Murcia ${ }^{4}$ and Christopher M Anderson ${ }^{1,2^{*}}$
}

\begin{abstract}
Background: Experimental autoimmune encephalomyelitis (EAE) is an animal model of multiple sclerosis characterized by entry of activated T cells and antigen presenting cells into the central nervous system and subsequent autoimmune destruction of nerve myelin. Previous studies revealed that non-selective inhibition of poly (ADP-ribose) polymerases (PARPs) 1 and 2 protect against neuroinflammation and motor dysfunction associated with EAE, but the role of the PARP-2 isoform has not yet been investigated selectively.

Results: EAE was induced in mice lacking PARP-2, and neurological EAE signs, blood-spine barrier (BSB) permeability, demyelination and inflammatory infiltration were monitored for 35 days after immunization. Mice lacking PARP-2 exhibited significantly reduced overall disease burden and peak neurological dysfunction. PARP-2 deletion also significantly delayed EAE onset and reduced BSB permeability, demyelination and central nervous system (CNS) markers of proinflammatory Th1 and Th17 T helper lymphocytes.

Conclusions: This study represents the first description of a significant role for PARP-2 in neuroinflammation and neurological dysfunction in EAE.

Keywords: CD4, CD11b, Demyelination, Experimental autoimmune encephalomyelitis, Neuroinflammation, Multiple sclerosis, PARP-1, PARP-2, Th1, Th17
\end{abstract}

\section{Introduction}

Multiple sclerosis (MS) affects up to $1 \%$ of the US population and $2 \%$ of Canadians [1,2], and is characterized by central nervous system (CNS) lymphocyte infiltration, autoimmune demyelination, axonal loss and neurodegeneration $[3,4]$. Experimental allergic encephalomyelitis (EAE) is an animal model that shares many features with human MS, including major histocompatibility complex (MHC)-linked susceptibility, female predominance, paralysis, ataxia,

\footnotetext{
* Correspondence: chris.anderson@med.umanitoba.ca

'Department of Pharmacology and Therapeutics, University of Manitoba and Division of Neurodegenerative Disorders, St Boniface Hospital Research,

Winnipeg, Canada

${ }^{2}$ Division of Neurodegenerative Disorders, St Boniface Hospital Research, Winnipeg, Canada

Full list of author information is available at the end of the article
}

increased cytokine production, and the presence of myelinreactive $\mathrm{T}$ cells [5].

Poly(ADP-ribose) polymerases (PARPs) comprise a superfamily of 18 enzymes involved in DNA repair and genomic stability [6,7]. PARP-1 and PARP-2 share the ability to catalyze poly ADP-ribosylation of target proteins [8] but PARP-1 accounts for about $90 \%$ of cellular poly ADP-ribosylation capacity [7,9]. Non-selective PARP-1/2 inhibitors reduce neuroinflammation and neurological dysfunction in rodent EAE [10-12] but it is not yet clear what the relative contributions of PARP-1 and PARP-2 are to this effect. Genetic PARP-1 loss of function in EAE has produced disparate results, both mitigating [13] and enhancing [14] EAE severity in mice. Our aim in the present work was to evaluate the role of PARP-2 deletion in EAE. 


\section{Methods}

All animal experiments were performed in accordance with guidelines of and approved by the Institutional Animal Care and Use Committee, University of Manitoba. EAE was induced in 8-week-old parp- $2^{-/-}$female mice [9] or wild-type littermate $\mathrm{C} 57 \mathrm{Bl} / 6$ controls by immunizing with myelin oligodendrocyte glycoprotein (MOG) $35-55$ and Freund's complete adjuvant (FCA) [15]. Two subcutaneous injections with $50 \mu \mathrm{g}$ of $\mathrm{MOG}_{35-55}$ emulsified in FCA containing $200 \mu \mathrm{g}$ of Mycobacterium tuberculosis were given on day 0 and 7 days after. Mice also received intraperitoneal pertussis toxin $(0.15 \mu \mathrm{g}$ in $100 \mu \mathrm{l}$ phosphate-buffered saline (PBS)) on day 0 and day 2 . Body weight and neurological score were measured daily. Mice were assigned a cumulative 14-point score [16], assessing function of tail and mobility of each front limb and each hind limb, up to 35 days after the second immunization. For tail function, a score of 0 is asymptomatic, while 1 refers to weakness and 2 is full paralysis. For the hind or forelimbs, each is assessed separately, with a score of 0 for no symptoms, 1 for weakness, 2 for limb dragging with limited movement, and 3 for full paralysis.

Permeability of the blood-spine barrier was determined at initiation of disease signs, as tracked by loss of $>1 \mathrm{~g}$ of body mass [17], by injecting mice with $100 \mu \mathrm{l}$ of $10 \%$ sodium fluorescein (Na-Fluor, intraperitoneal administration) and monitoring fluorescence in spinal homogenates after 10 minutes.

For histology and immunofluorescence, cervical and thoracic cord segments were snap frozen and sectioned $(10 \mu \mathrm{m})$ at peak disease scores, determined empirically for each mouse as the fifth day after initial disease signs $($ score $>0)$. Tissue was stained with hematoxylin and eosin $(\mathrm{H} \& \mathrm{E})$ to detect CNS inflammatory infiltrates, or solochrome cyanin to assess myelination, and qualitative assessment of each parameter was performed as described previously [16]. All assessments were made by a blinded observer. For immunohistochemistry, primary antibodies were rat anti-CD11b (EMD-Millipore, Billerica, MA, USA), rat anti-CD4 (BD Biosciences, San Diego, CA, USA), rabbit anti-ROR- $\gamma$ T (Abcam, Cambridge, MA, USA) and mouse anti-T-bet (Abcam). Primary antibodies were applied overnight at $4^{\circ} \mathrm{C}$. Secondary antibodies were either Alexa Fluor 488 or 560 conjugates (Life Technologies, Carlsbad, CA, USA).

Non-parametric two-tailed Mann-Whitney tests were used to analyze the differences in EAE neurological signs (two groups). Kruskal-Wallis non-parametric analysis of variance (ANOVA) with Dunn's multiple comparisons test was used for qualitative assessment of clinical scoring with more than two groups. For all other comparisons, one-way ANOVA followed by Student Newman-Keuls multiple comparison test was used.

\section{Results}

Silencing PARP-2 protects neurological function and reduces blood-spine barrier permeability in mouse EAE

EAE was induced in PARP-2 null mice and wild-type littermate $\mathrm{C} 57 \mathrm{Bl} / 6$ controls. Body mass and neurological signs of disease were monitored for 35 days after the second $\mathrm{MOG}_{35-55}$ immunization. Figure $1 \mathrm{~A}$ shows the temporal disease profile for $\mathrm{C} 57 \mathrm{Bl} / 6(\mathrm{n}=9)$ and PARP-2 null mice $(\mathrm{n}=6)$. PARP-2 deletion reduced the 35 day total disease burden (aggregate daily score per 35 EAE days) from $4.8 \pm$ 1.5 to $2.6 \pm 1.0$ and the peak neurological severity score from $9.6 \pm 1.1$ to $6.9 \pm 2.2$ (Figure 1B). PARP-2 deletion also significantly increased the number of days required to reach disease peak from $8.7 \pm 3.5$ days to $13.4 \pm 2.7$ days after the second immunization (Figure 1C).

Blood-spine barrier (BSB) permeability was assessed immediately following large weight loss $(1.89 \pm 0.66 \mathrm{~g}, \mathrm{n}=$ 13) corresponding to peak transient BSB permeability [18]. Following intraperitoneal Na-Fluor administration (10 minutes), spinal fluorescence increased tenfold in EAE mice $(\mathrm{n}=4)$ compared to vehicle-treated (sham, $\mathrm{n}=3$ ) controls (Figure 1D). BSB permeability was also significantly enhanced by PARP-2 null EAE mice $(n=4)$, compared to the PARP-2 null sham group $(n=4)$, however, the magnitude of this enhancement was considerably smaller (3.7-fold) than observed in wild-type mice.

\section{PARP-2 deletion reduces spinal inflammatory cell infiltration and demyelination in $\mathrm{EAE}$}

Mice were sacrificed at peak EAE score, designated as day 5 after each individual animal displayed a neurological score greater than 0 , and cervical and thoracic spinal sections were stained with H\&E. Wild-type EAE mice exhibited extensive perivascular parenchymal cell infiltration, relative to sham animals (Figure 2A,B), which was reduced by PARP-2 deletion (Figure 2C). Quantification in Figure 2D shows that central infiltration in wild-type mice $(\mathrm{n}=7)$ is statistically reduced by the parp- $2^{-/-}$genotype $(n=4)$.

We next investigated whether there was proinflammatory $\mathrm{T}$ lymphocyte infiltration. Parenchymal $\mathrm{CD} 4^{+}$cell counts per $\mathrm{mm}^{2}$ increased from $36.1 \pm 12.8$ to $400.1 \pm 63.0$ in spinal cord sections of EAE mice $(n=7)$, relative to sham controls $(\mathrm{n}=3)$. PARP-2 deletion significantly reduced EAE $\mathrm{CD}^{+}$counts to $146.7 \pm 12.8(\mathrm{n}=4$; Figure $3 \mathrm{~A}-\mathrm{C})$. To determine effects of PARP- 2 on proinflammatory Th1 and Th17 T helper cell phenotypes, coimmunofluorescence experiments were performed for CD4 and either T-bet (Th1) [19] or ROR- $\gamma \mathrm{T}$ (Th17) [20]. EAE increased the number of $\mathrm{CD}^{+} /$T-bet $^{+}$cells from $3.9 \pm 1.6$ in sham cords $(\mathrm{n}=3)$ to $94.8 \pm 14.6$ in $\operatorname{EAE}(n=7)$. Elimination of PARP-2 reduced this to $21.7 \pm 2.8 \mathrm{cell} / \mathrm{mm}^{2}(\mathrm{n}=4$; Figure 3D). Similarly, EAE increased $\mathrm{CD}^{+} / \mathrm{ROR}-\gamma \mathrm{T}^{+}$cell counts from $1.6 \pm 0.8$ in sham controls to $43.2 \pm 6.9$ in EAE in a manner dependent on PARP-2, as deletion of PARP-2 reduced EAE 

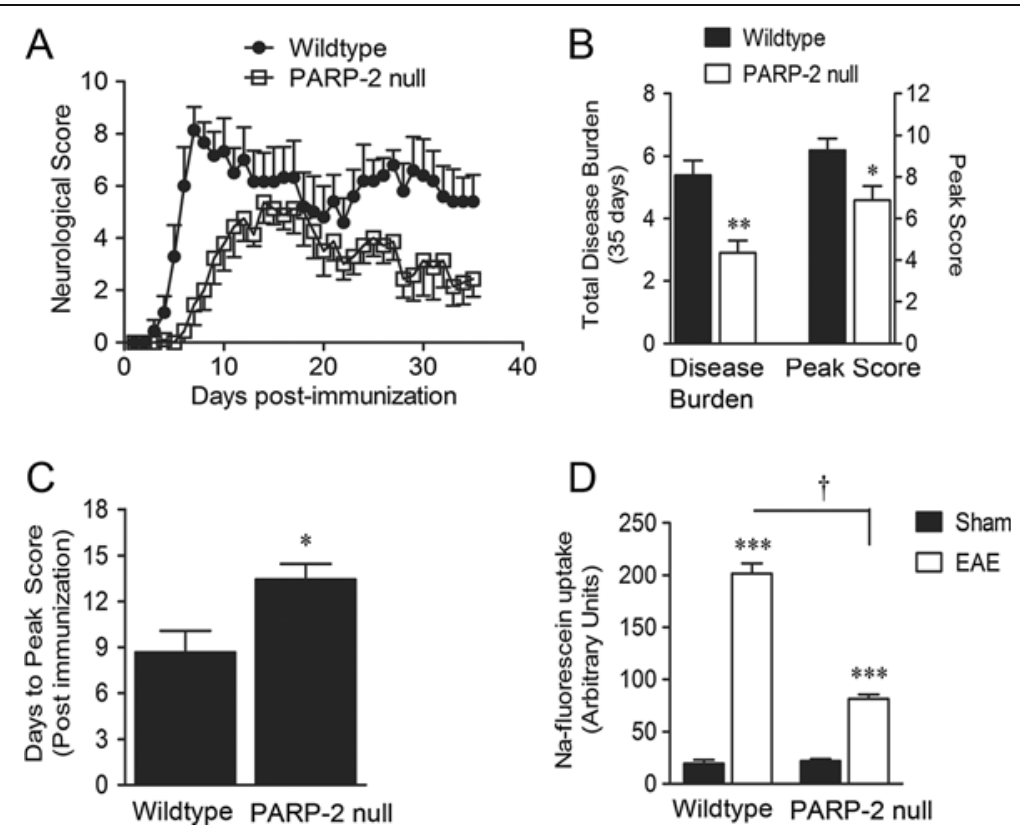

Figure 1 Deletion of poly(ADP-ribose) polymerase 2 (PARP-2) protects neurological function and blood-spine barrier integrity in experimental autoimmune encephalomyelitis (EAE) mice. (A) PARP-2 null and littermate C57BI/6 mice were immunized with myelin oligodendrocyte glycoprotein (MOG) ${ }_{35-55}$ and neurological signs of EAE were scored for 35 days. (A) Daily neurological scoring profile for wild-type and PARP-2 null EAE mice. (B) Total disease burden (summed score/35 days) and peak neurological score were significantly reduced by PARP-2 deletion. (C) The number of days required to reach first peak disease score was significantly increased by PARP-2 deletion. (D) Spinal:serum fluorescence was measured following sodium fluorescein (Na-Fluor) administration intraperitoneally just prior to peak disease neurological score. The magnitude of the EAE-induced ratio increase was significantly less in PARP-2 mice than in wild-type animals. Values are expressed as mean \pm SEM ( $n=6$ to 9). (B,C ${ }^{*} P<0.05$, using non-parametric two-tailed Mann-Whitney test. (D) ${ }^{* *} P<0.001$ compared to respective sham control, $+P<0.001$ as indicated using one-way analysis of variance (ANOVA) followed by Student Newman-Keuls multiple comparisons test.

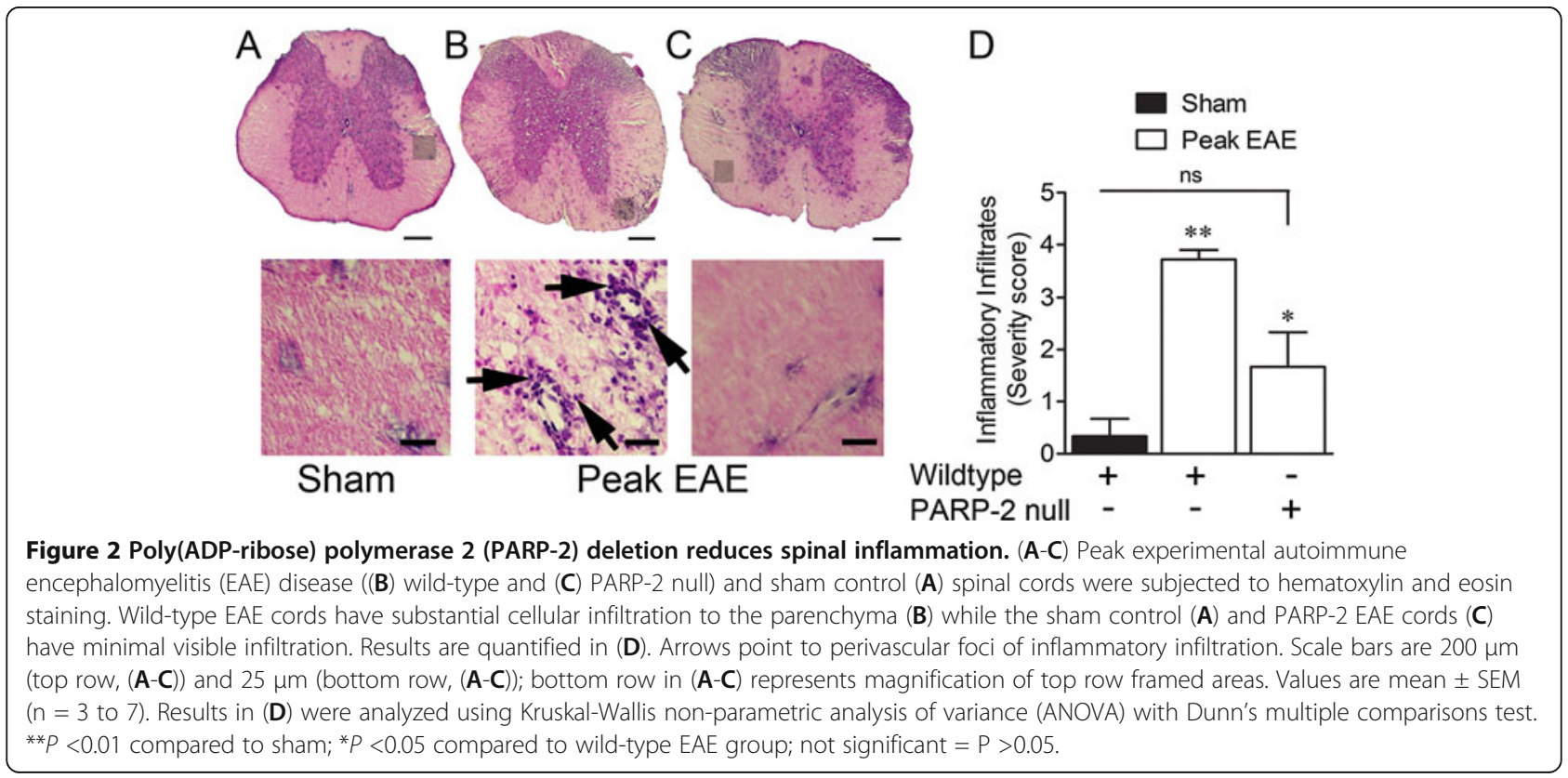



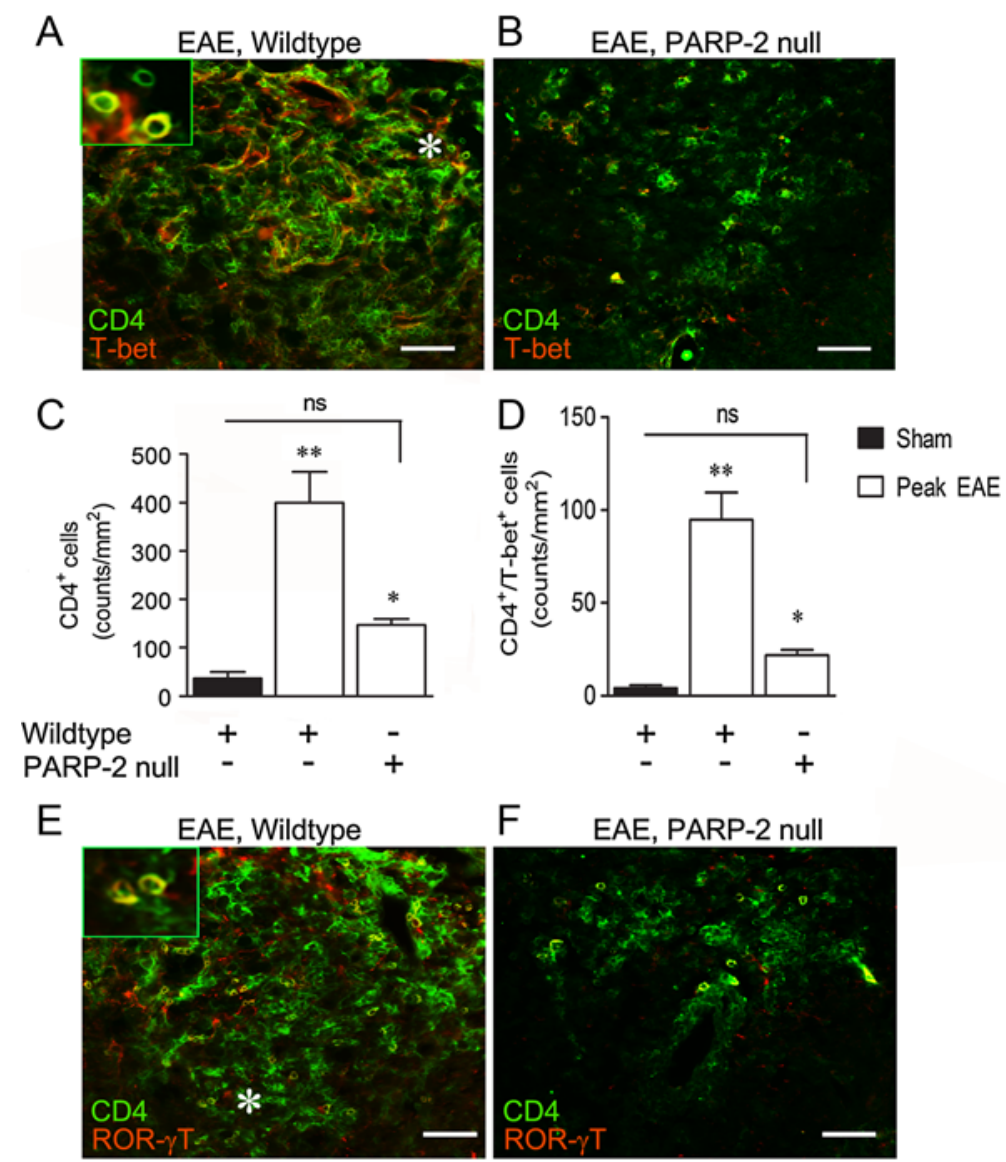

F $\quad$ EAE, PARP-2 null
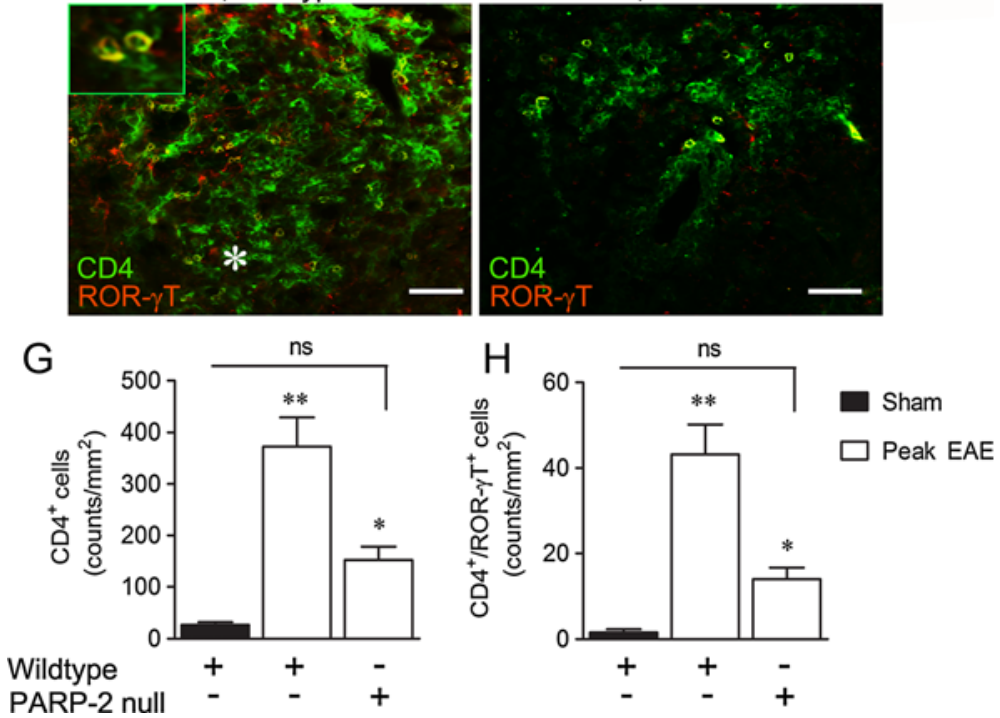

Figure 3 Poly(ADP-ribose) polymerase 2 (PARP-2) deletion reduces Th1 and Th17 T helper cell infiltration in experimental autoimmune encephalomyelitis (EAE) mice spinal cords. Sham control and peak EAE spinal cords from wild-type and PARP-2 null mice were subjected to coimmunofluorescence staining using antibodies for CD4 and T-bet (A-D) or CD4 and ROR- $\mathbf{T}^{\top}(\mathbf{E}-\mathrm{H})$. Widespread CD4 ${ }^{+}$immunoreactivity (IR) was observed in wild-type EAE spinal sections (B), approximately 24\% of which was also T-bet ${ }^{+}$. The inset shows a magnified image of the area directly to the right of the asterisk symbol (*) and illustrates cellular colocalization (yellow). (B) Representative image of reduced $\mathrm{CD} 4^{+}$and $^{\mathrm{CD}} 4^{+} / \mathrm{T}_{\text {-bet }}{ }^{+}$infiltration in PARP-2 null EAE cords. (C,D) Quantifications of $\mathrm{CD}^{+}$and $\mathrm{CD} 4^{+} / T$-bet ${ }^{+}$cell counts and indicate a significant reduction of each with elimination of PARP-2. In (E), 12\% of $C D^{+}$cells were also ROR- $\mathrm{Y}^{+}$. The inset in (E) shows a magnified image of the area directly to the left of the asterisk symbol $\left(^{*}\right)$. (F) Representative image of reduced $\mathrm{CD}^{+}$and $\mathrm{CD} 4^{+} / \mathrm{ROR}_{-} \mathrm{T}^{+}$infiltration in PARP-2 null EAE cords. (G,H) Quantifications of $\mathrm{CD} 4^{+}$and $\mathrm{CD} 4^{+} / \mathrm{ROR}_{-} \mathrm{T}^{+}$ cell counts and indicate a significant reduction of each with elimination of PARP-2. Results in $(\mathbf{C}, \mathbf{D}, \mathbf{G}, \mathbf{H})$ are expressed as means \pm SEM and were analyzed using one-way analysis of variance (ANOVA) with the Student Newman-Keuls multiple comparison test. ${ }^{*} P<0.01$ compared to sham; ${ }^{*} P<0.05$ compared to wild-type EAE; not significant $=P>0.05$. Scale bars are $25 \mu \mathrm{m}$.

counts to $14.0 \pm 2.7$ (Figure 3E-H). Salutary effects of PARP-2 deletion on spinal microglial/macrophage CD11b accumulation in EAE were also observed. Spinal regions with H\&E stain-verified inflammation exhibited widespread
CD11b ${ }^{+}$immunoreactivity (IR) in EAE (Figure 4A, B) that was PARP-2 dependent (Figure 4C, D).

Multifocal areas of reduced solochrome cyanin intensity revealed widespread, diffuse demyelination in wild- 


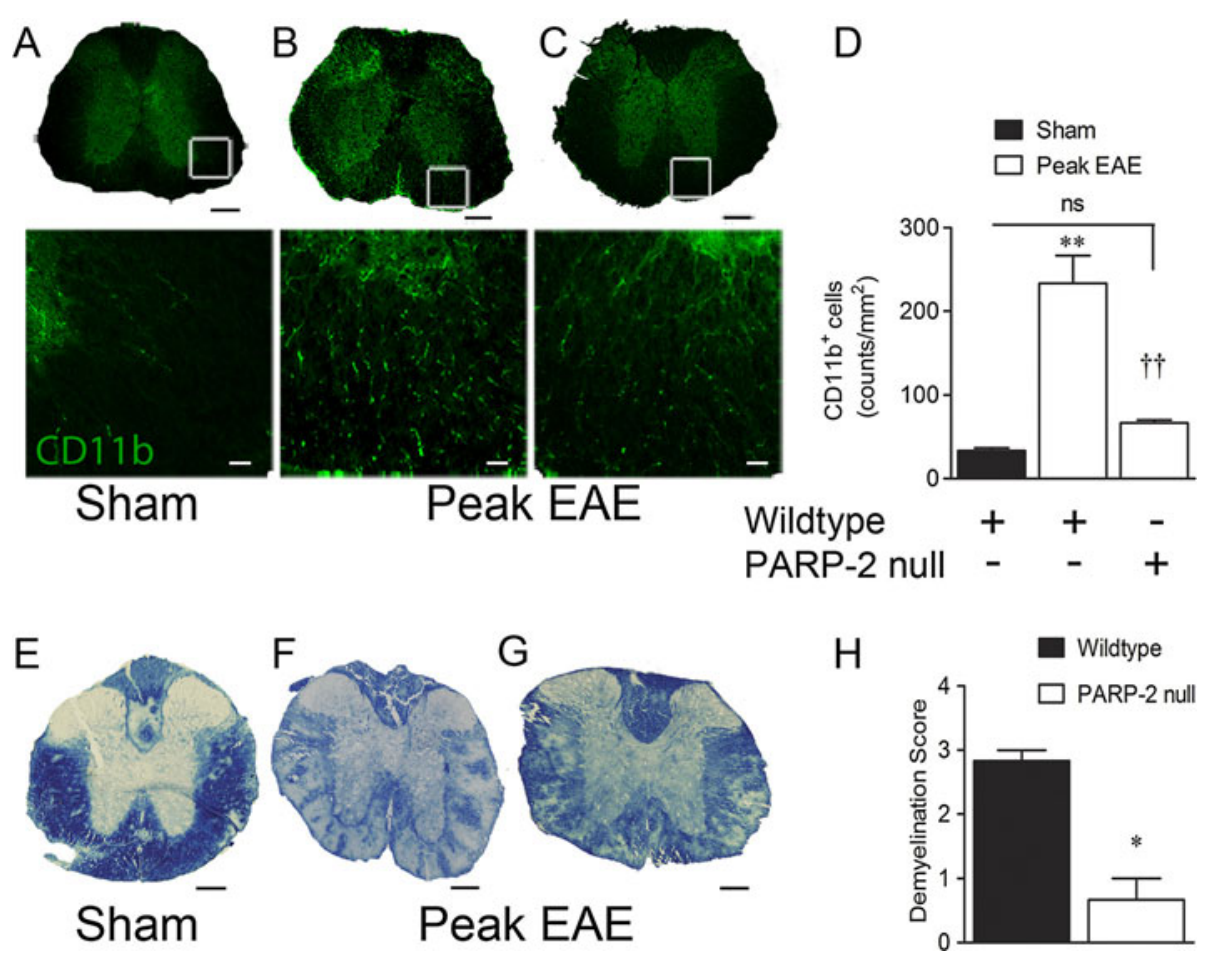

Figure 4 Poly(ADP-ribose) polymerase 2 (PARP-2) contributes to macrophage accumulation and spinal demyelination in experimental autoimmune encephalomyelitis (EAE). CD11b immunofluorescence was used to detect macrophage/microglial cells activated by EAE. (B) Representative images illustrating elevated $C D 11 b^{+}$immunoreactivity in wild-type EAE spinal sections compared with sham sections (A). PARP-2 deletion reduced CD11b IR $(\mathbf{C})$. Results were quantified in (D). Values are mean \pm SEM ( $n=3$ to 7$)$ and were analyzed using one-way analysis of variance (ANOVA) with the Student Newman-Keuls multiple comparison test. ${ }^{*} P<0.01$ compared to sham; $+\uparrow P<0.01$ compared to wild-type EAE; not significant $=P>0.05$. Scale bars are $200 \mu \mathrm{m}$ (top row, $\mathbf{A}-\mathbf{C}$ ) and $25 \mu \mathrm{m}$ (bottom row, $(\mathbf{A}-\mathbf{C})$ ). Bottom row represents magnification of framed areas in the top row. Demyelination was assessed in peak disease EAE mice by staining with solochrome cyanin. Wild-type sham spinal cord white matter is thoroughly myelinated, as indicated by deep blue staining (E). Wild-type EAE spinal cords exhibit extensive areas of demyelination (F). EAE in PARP-2 null spinal cords produced only a low degree of demyelination (G) relative to wild-type controls. Results are quantified in $(\mathbf{H})$ and show a significant reduction in demyelination of PARP-2 null cords compared to wild-type cords in EAE. Scale bars are $200 \mu \mathrm{m}$. Values are mean \pm SEM $\left(n=3\right.$ to 6). Results in $(\mathbf{H})$ were analyzed using non-parametric two-tailed Mann-Whitney test. ${ }^{*} P<0.05$ compared to wild-type EAE group.

type EAE mice (Figure 4E, F) that was significantly reduced in PARP-2 null EAE mice (Figure 4G, H). These results support a role for PARP-2 in EAE-induced spinal demyelination in mice.

\section{Discussion}

We addressed the role of PARP-2 in EAE using parp-2 ${ }^{-/-}$ mice. Embryonic deletion of PARP-2 protected EAE mice from neuroinflammation and neurological dysfunction. Overall, parp $-2^{-/-}$mice had significantly lower total disease burden over the 35 day period studied, compared to wild-type $\mathrm{C} 57 \mathrm{Bl} / 6$ controls. Peak EAE neurological scores were also significantly reduced by eliminating PARP-2. In practical terms, this peak protection means that hindlimb paralysis and tail/forelimb weakness observed in wild-type EAE mice were prevented in parp- $2^{-/-}$mice, which experienced average maximal scores corresponding to hindlimb weakness but no paralysis or tail and forelimb effects. In addition, while we found a discernable peak EAE score in parp- $2^{-/-}$mice, the peak was shifted temporally further away from EAE induction, compared to wild-type mice, representing a 50\% delay in EAE peak effect. We also evaluated BSB permeability and series of markers for EAE neuroinflammation. The parp- $2^{-/-}$genotype significantly reduced EAE-induced BSB permeability and spinal distri-

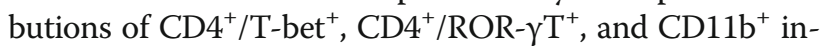
flammatory infiltrates, as well as demyelination in the cervical and thoracic spinal cord. Taken together, these data demonstrate that embryonic deletion of PARP-2 protects nerve myelin and motor performance while reducing neuroinflammation associated with EAE.

Our observation that PARP-2 deletion is protective in EAE raises the possibility that PARP inhibitors shown previously to have beneficial effects in EAE are effective at least partially by interfering with the PARP-2 isoform. In agreement, PJ34 [11], 6(5H)-phenanthridinone (PHE) [10] and 5-aminoisoquinolinone (5-AIQ) [13] improved EAE outcomes and all compete for the $\mathrm{NAD}^{+}$binding site of 
both PARP-1 and PARP-2 [21-23], and we (data not shown) and others [14] found that PARP-1 deletion is not protective but rather enhances peak disease severity. However, PARP-1 deletion reduced neuroinflammation and improved EAE outcome in another EAE model [13], making categorical conclusions about the relative roles of PARP-1 and PARP-2 in EAE difficult at this point. It should be noted that embryonic deletion of PARP isoforms prior to EAE could have different effects on EAE outcomes than adult pharmacological or genetic loss of function. For example, parp- $1^{-/-}$mice have higher numbers of regulatory and EAE-activated $\mathrm{CD} 4^{+} / \mathrm{CD}^{+} \mathrm{T}$ lymphocytes compared with wild-type animals $[14,24]$ and

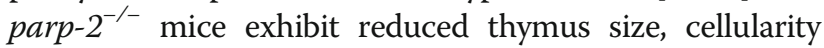
and numbers of peripheral $\mathrm{T}$ cell precursor thymocytes [25]. In both of these cases, there could be a phenotypic predisposition to a different immune response prior to EAE induction that would not be present in wild-type animals. Further work employing models in which PARP isoforms can be selectively inhibited or deleted just prior to or during EAE (conditional knockouts) are required to precisely delineate the neuroinflammatory roles of PARP1 and PARP-2, and to determine the potential usefulness of these isoforms as therapeutic targets in adult EAE.

\section{Conclusions}

The current study implicates PARP-2 in neuroinflammation and neurological signs of EAE for the first time. Identification of a selective role for PARP-2 in EAE progression establishes a novel therapeutic target of interest for neuroinflammation and MS.

\section{Competing interests}

The authors report no conflicts of interest.

\section{Authors' contributions}

AK was responsible for inducing EAE, animal care, keeping record of daily weights, neurological scoring of the EAE animals, manuscript preparation and performing assessments of blood-spine barrier permeability, histology, and statistical analysis. PL carried out immunohistochemical analysis. MBC prepared tissues for histology and immunohistochemistry. JLS assisted AK in preparing myelin oligodendrocyte glycoprotein, inducing EAE and in transcardiac perfusion. BAD performed the preliminary EAE experiments and was involved in conceptual design. TMK contributed significantly to the intellectual development of the project. GM provided the PARP-2 knockout strain for performing EAE studies. CMA conceived and designed the study, supervised all personnel and helped to draft the manuscript. All authors read and approved the final manuscript.

\section{Acknowledgements \\ Direct research costs were supported by the St Boniface Hospital and Research Foundation and the Multiple Sclerosis Society of Canada. AK and PL were supported by fellowships from the Manitoba Health Research Council and the St Boniface Hospital and Research Foundation. JLS was supported by a Doctoral Research Award from the Canadian Institutes of Health Research. The authors thank Dr Sam Kung for helpful comments.}

\section{Author details}

'Department of Pharmacology and Therapeutics, University of Manitoba and Division of Neurodegenerative Disorders, St Boniface Hospital Research, Winnipeg, Canada. ${ }^{2}$ Division of Neurodegenerative Disorders, St Boniface
Hospital Research, Winnipeg, Canada. ${ }^{3}$ Department of Internal Medicine, University of Manitoba, Winnipeg, Canada. ${ }^{4}$ Department 'Intégrité du Génome' de I'UMR 7175, École Supérieure de Biotechnologie de Strasbourg, Illkirch Cedex, France.

Received: 16 October 2012 Accepted: 10 April 2013 Published: 22 April 2013

\section{References}

1. Anderson DW, Ellenberg JH, Leventhal CM, Reingold SC, Rodriguez M, Silberberg DH: Revised estimate of the prevalence of multiple sclerosis in the United States. Ann Neurol 1992, 31:333-336.

2. Girouard N, Soucy N: Patient considerations in the management of multiple sclerosis: development and clinical utility of oral agents. Patient Prefer Adherence 2011, 5:101-108.

3. Neuhaus O, Archelos JJ, Hartung HP: Immunomodulation in multiple sclerosis: from immunosuppression to neuroprotection. Trends Pharmacol Sci 2003, 24:131-138.

4. Bjartmar C, Trapp BD: Axonal and neuronal degeneration in multiple sclerosis: mechanisms and functional consequences. Curr Opin Neurol 2001, 14:271-278.

5. Link H, Xiao BG: Rat models as tool to develop new immunotherapies. Immunol Rev 2001, 184:117-128.

6. Ame JC, Spenlehauer C, de Murcia G: The PARP superfamily. BioEssays 2004, 26:882-893.

7. Huber A, Bai P, de Murcia JM, de Murcia G: PARP-1, PARP-2 and ATM in the DNA damage response: functional synergy in mouse development. DNA Repair (Amst) 2004, 3:1103-1108.

8. Ménissier de Murcia J, Ricoul M, Tartier L, Niedergang C, Huber A, Dantzer F, Schreiber V, Amé JC, Dierich A, LeMeur M, Sabatier L, Chambon P, de Murcia G: Functional interaction between PARP-1 and PARP-2 in chromosome stability and embryonic development in mouse. EMBO J 2003, 22:2255-2263.

9. Schreiber V, Ame JC, Dolle P, Schultz I, Rinaldi B, Fraulob V, Menissier-de Murcia J, de Murcia G: Poly(ADP-ribose) polymerase-2 (PARP-2) is required for efficient base excision DNA repair in association with PARP-1 and XRCC1. J Biol Chem 2002, 277:23028-23036.

10. Chiarugi $A$ : Inhibitors of poly(ADP-ribose) polymerase- 1 suppress transcriptional activation in lymphocytes and ameliorate autoimmune encephalomyelitis in rats. Br J Pharmacol 2002, 137:761-770.

11. Scott GS, Kean RB, Mikheeva T, Fabis MJ, Mabley JG, Szabo C, Hooper DC: The therapeutic effects of PJ34 [N-(6-oxo-5,6-dihydrophenanthridin-2-yl)$\mathrm{N}, \mathrm{N}$-dimethylacetamide. $\mathrm{HCl}$, a selective inhibitor of poly(ADP-ribose) polymerase, in experimental allergic encephalomyelitis are associated with immunomodulation. J Pharmacol Exp Ther 2004, 310:1053-1061.

12. Cavone L, Aldinucci A, Ballerini C, Biagioli T, Moroni F, Chiarugi A: PARP-1 inhibition prevents CNS migration of dendritic cells during $E A E$, suppressing the encephalitogenic response and relapse severity. Mult Scler 2011, 17:794-807.

13. Farez MF, Quintana FJ, Gandhi R, Izquierdo G, Lucas M, Weiner HL: Toll-like receptor 2 and poly(ADP-ribose) polymerase 1 promote central nervous system neuroinflammation in progressive EAE. Nat Immunol 2009, 10:958-964

14. Selvaraj V, Soundarapandian MM, Chechneva O, Williams AJ, Sidorov MK, Soulika AM, Pleasure DE, Deng W: PARP-1 deficiency increases the severity of disease in a mouse model of multiple sclerosis. J Biol Chem 2009, 284:26070-26084.

15. Brundula V, Rewcastle NB, Metz LM, Bernard CC, Yong WW: Targeting leukocyte MMPs and transmigration: minocycline as a potential therapy for multiple sclerosis. Brain 2002, 125:1297-1308.

16. Giuliani F, Metz LM, Wilson T, Fan Y, Bar-Or A, Yong WW: Additive effect of the combination of glatiramer acetate and minocycline in a model of MS. J Neuroimmunol 2005, 158:213-221.

17. Touil T, Ciric B, Ventura E, Shindler KS, Gran B, Rostami A: Bowman-Birk inhibitor suppresses autoimmune inflammation and neuronal loss in a mouse model of multiple sclerosis. J Neurol Sci 2008, 271:191-202.

18. Sanna V, Di Giacomo A, La Cava A, Lechler RI, Fontana S, Zappacosta S, Matarese G: Leptin surge precedes onset of autoimmune encephalomyelitis and correlates with development of pathogenic T cell responses. J Clin Invest 2003, 111:241-250. 
19. Szabo SJ, Kim ST, Costa GL, Zhang X, Fathman CG, Glimcher LH: A novel transcription factor, T-bet, directs Th1 lineage commitment. Cell 2000, 100:655-669.

20. Ivanov II, McKenzie BS, Zhou L, Tadokoro CE, Lepelley A, Lafaille JJ, Cua DJ, Littman DR: The orphan nuclear receptor RORgammat directs the differentiation program of proinflammatory $\mathrm{IL}-17^{+} \mathrm{T}$ helper cells. Cell 2006, 126:1121-1133.

21. Iwashita A, Tojo N, Matsuura S, Yamazaki S, Kamijo K, Ishida J, Yamamoto H, Hattori K, Matsuoka N, Mutoh S: A novel and potent poly(ADP-ribose) polymerase-1 inhibitor, FR247304 (5-chloro-2-[3-(4-phenyl-3,6-dihydro-1 (2H)-pyridinyl)propyl]-4(3H)-quinazo linone), attenuates neuronal damage in in vitro and in vivo models of cerebral ischemia. J Pharmacol Exp Ther 2004, 310:425-436.

22. Jelezcova E, Trivedi RN, Wang XH, Tang JB, Brown AR, Goellner EM, Schamus S, Fornsaglio JL, Sobol RW: Parp1 activation in mouse embryonic fibroblasts promotes Pol beta-dependent cellular hypersensitivity to alkylation damage. Mutat Res 2010, 686:57-67.

23. Sunderland PT, Dhami A, Mahon MF, Jones LA, Tully SR, Lloyd MD, Thompson AS, Javaid H, Martin NM, Threadgill MD: Synthesis of 4-alkyl-, 4-aryl- and 4-arylamino-5-aminoisoquinolin-1-ones and identification of a new PARP-2 selective inhibitor. Org Biomol Chem 2011, 9:881-891.

24. Nasta F, Laudisi F, Sambucci M, Rosado MM, Pioli C: Increased Foxp $3^{+}$ regulatory T cells in poly(ADP-Ribose) polymerase-1 deficiency. J Immunol 2010, 184:3470-3477.

25. Yélamos J, Monreal Y, Saenz L, Aguado E, Schreiber V, Mota R, Fuente T, Minguela A, Parrilla P, de Murcia G, Almarza E, Aparicio P, Ménissier-de Murcia J: PARP-2 deficiency affects the survival of $\mathrm{CD}^{+} \mathrm{CD}^{+}$doublepositive thymocytes. EMBO J 2006, 25:4350-4360.

doi:10.1186/1742-2094-10-49

Cite this article as: Kamboj et al:: Poly(ADP-ribose) polymerase 2 contributes to neuroinflammation and neurological dysfunction in mouse experimental autoimmune encephalomyelitis. Journal of Neuroinflammation 2013 10:49.

\section{Submit your next manuscript to BioMed Central and take full advantage of:}

- Convenient online submission

- Thorough peer review

- No space constraints or color figure charges

- Immediate publication on acceptance

- Inclusion in PubMed, CAS, Scopus and Google Scholar

- Research which is freely available for redistribution 